\title{
MINKOWSKI SPACETIME AND LORENTZ INVARIANCE: THE CART AND THE HORSE OR TWO SIDES OF A SINGLE COIN? ${ }^{1}$
}

\author{
Pablo Acuña \\ Instituto de Filosofía, Pontificia Universidad Católica de Valparaíso \\ pablo.acuna.1@pucv.cl
}

\begin{abstract}
Michel Janssen and Harvey Brown have driven a prominent recent debate concerning the direction of an alleged arrow of explanation between Minkowski spacetime and Lorentz invariance of dynamical laws in special relativity. In this article, I critically assess this controversy with the aim of clarifying the explanatory foundations of the theory. First, I show that two assumptions shared by the parties - that the dispute is independent of issues concerning spacetime ontology, and that there is an urgent need for a constructive interpretation of special relativity-are problematic and negatively affect the debate. Second, I argue that the whole discussion relies on a misleading conception of the link between Minkowski spacetime structure and Lorentz invariance, a misconception that in turn sheds more shadows than light on our understanding of the explanatory nature and power of Einstein's theory. I state that the arrow connecting Lorentz invariance and Minkowski spacetime is not explanatory and unidirectional, but analytic and bidirectional, and that this analytic arrow grounds the chronogeometric explanations of physical phenomena that special relativity offers.
\end{abstract}

Keywords: special relativity, Lorentz invariance, Minkowski spacetime, explanation, theories of principle, constructive theories, spacetime ontology.

\section{INTRODUCTION}

Over the last decade, an interpretive debate about the explanatory nature of Einstein's special theory of relativity has developed. Michel Janssen and Harvey Brown have driven it (Janssen 1995, 2002a, 2002b, 2009; Balashov and Janssen 2003; Brown 2003, 2005a, 2005b; Brown \& Pooley 2001, 2006), and it has become a much-discussed issue in the philosophy of physics literature (e.g., Norton 2008, Frisch 2011, Van Camp 2011, Dorato \& Felline 2010, Felline 2011). The point in dispute concerns the direction of an alleged arrow of explanation between Minkowski spacetime structure and Lorentz invariance of physical laws: they argue about which is the cart and which is the horse. Harvey Brown affirms that Lorentz invariance explains the Minkowski structure of spacetime, whereas Janssen claims that the arrow of explanation points in the opposite direction, i.e., from Minkowski structure to Lorentz invariance:

Our central disagreement [...] is a dispute about the direction of the arrow of explanation connecting the symmetries of Minkowski spacetime and the Lorentz-invariance of the dynamical laws governing systems in Minkowski spacetime. I argue that the spacetime symmetries are the explanans and that the Lorentz invariance of the various laws is the explanandum. Brown argues that it is the other way around. (Janssen 2009, 29)

The first goal of this article is to show that the dispute relies on a misleading overinterpretation of the relation between Minkowski structure and Lorentz invariance in special relativity, which in turn results in a misconception of the explanatory nature of Einstein's theory. I point out two problematic issues underlying the debate that have a significant negative import on its overall evaluation. More

\footnotetext{
${ }^{1}$ This paper has appeared in published form in Studies In History and Philosophy of Modern Physics, 55: 1-12. For quoting purposes, please refer to the published version.
} 
precisely, despite the authors' explicit comments to the contrary, I show that the discussion is knotted with the question of the ontology of spacetime: Janssen's view is connected to a form of substantivalism, whereas Brown's view assumes a form of relationism. I also show that the urgent demand for a constructive interpretation of special relativity that Janssen and Brown argue for is unjustified and unnecessary, and that it actually obscures our understanding of the explanatory nature and power of the theory.

Second, I argue that rather than a debate about what is the cart and what is the horse, the discussion between Brown and Janssen is an instance of the dispute about what was first, the egg or the chicken. I claim that Minkowski structure and Lorentz invariance need not be conceived in such a way that one must explain the other. Instead, they are better understood as the two sides of a single coin: the arrow between them is not explanatory, but analytic (in a sense that I explain below). Furthermore, I argue that this conception of the link between Lorentz invariance and Minkowski chronogeometry, unlike the conception that motivates the Janssen-Brown debate, sheds light on our understanding of the explanatory nature and power of special relativity. I claim that the aforementioned analytic connection between Minkowski structure and Lorentz grounds the explanatory foundations of Einstein's theory.

The structure of this article is the following. In section 2, I provide an overview of the debate, paying special attention to the aspects that are most relevant for my critical assessment. In section 3, I underscore two crucial assumptions endorsed by Brown and Janssen - that the discussion is independent of questions about spacetime ontology, and that the arrow of explanation at stake must be constructiveand I ponder about the justification that the authors offer (and implicitly assume) for them. In section 4, I show that both these assumptions are problematic and unjustified, thus tainting the whole debate. In section 5, I argue that Minkowski structure and Lorentz invariance are analytically linked, and that this link - that is not itself explanatory - clarifies the source of the chronogeometric explanations of physical phenomena that special relativity provides. In section 6, I conclude and value the lessons that we can draw from Janssen's and Brown's arguments in the light of my present proposal.

\section{THE JANSSEN-BROWN DEBATE}

The debate at issue concerns the direction of an alleged arrow of explanation between the structure of Minkowski spacetime and the Lorentz invariance of physical laws. Brown states that the latter explains the former, whereas Janssen asserts that the explanatory arrow goes from Minkowski structure to Lorentz invariance. In this section, I analyze both stances in turn.

\subsection{BROWN'S INTERPRETATION}

According to Harvey Brown, it is a widespread view in foundational studies on special relativity that dynamical aspects of the theory are explained by the structure of Minkowski spacetime. As an example, Brown $(2005 a, 24)$ quotes a passage in which Graham Nerlich provides an explanation of inertial motion given by the shape of Minkowski spacetime. Nerlich states that since a particle cannot be 'aware' of how all the rest of the objects behave, the fact that when the particle moves freely it follows a characteristic trajectory cannot be explained in relational terms. Thus, Nerlich argues, the physical underpinning of inertial motion must rely on the affine structure of Minkowski spacetime:

Without the affine structure there is nothing to determine how the [free] particle trajectory should lie. It has no antennae to tell it where other objects are, even if there were other objects [...]. It is because spacetime has a certain shape that world lines lie as they do. (Nerlich 1976, 264) 
Brown proposes two arguments against this view. First, he states that this line of thought is highly problematic from an ontological standpoint. Even if we take for granted that there is a self-standing spacetime endowed with a specific structure, it is deeply mysterious how physical objects can get to 'know' this structure, so that they can behave accordingly. Brown claims that Nerlich's view amounts to state that freely moving objects follow the ruts and grooves of spacetime, just as an iron ball follows the groove of an inclined plane. However, since spacetime is not a physical object like an inclined plane, there cannot be any guiding friction, so it is not comprehensible how spacetime can determine the trajectory of a freely moving object ${ }^{2}$.

Brown's second argument against the standard view is of a more logical vein. Even if we assume that there is a self-standing spacetime with a specific structure, it does not follow from this assumption that the dynamical symmetries of physical laws mirror such a structure:

As a matter of logic alone, if one postulates spacetime structure as a self-standing, autonomous element in one's theory, it need have no constraining role on the form of the laws governing the rest of the theory's models. So how is its influence supposed to work? Unless this question is answered, spacetime cannot be taken to explain the Lorentz covariance of the dynamical laws. (Brown \& Pooley 2006, 84)

Brown \& Pooley are certainly right in this point. There is a historical example that illustrates that there is no guaranteed correspondence between spacetime structure and dynamical symmetries, namely, Lorentz's ether theory. The spacetime structure postulated by this theory is Newtonian, whereas the dynamical symmetries of physical laws are given by the Lorentz transformations (see Acuña 2014; Janssen 1995, chapter 3).

Considering the ontological and logical difficulties of the 'standard' view, Brown asserts that it is much more natural and plausible to conceive the link between Minkowski spacetime structure and Lorentz invariance in such a way that the latter explains the former. That is, Brown asserts that special relativistic spacetime has the structure it has because the symmetries of the dynamical laws that govern physical systems are the Lorentz transformations: "the appropriate structure is Minkowski geometry precisely because the laws of physics [...] are Lorentz covariant" (Brown \& Pooley 2006, 80).

A very important feature in Brown's interpretation, usually overlooked in the related literature, is that it is, in a sense, provisional. The physical underpinning of Lorentz invariance is not explicitly accounted for-in the current state of science-by a fundamental theory of matter. The most natural candidate to fulfill this task is quantum theory. However, in quantum field theory the Lorentz invariance of fundamental dynamical laws is written in by hand, it is not a result (cf. Hagar \& Hemmo 2013). Thus, quantum theory, in its current state of development, does not provide an explanation of Lorentz invariance at a fundamental level. This is why Brown describes his interpretation as truncated: although we must understand Lorentz invariance as the explanans and Minkowski structure as the explanandum, the

\footnotetext{
${ }^{2}$ Nerlich (2010) replies that Brown's is a parodical misrepresentation of his views. He openly rejects that spacetime causally explains inertial motion. Rather, Nerlich states that the kind of explanation involved is geometrical. He points out that straight trajectories, defined by the affine structure of Newtonian spacetime, constitute the default (force-free, zero acceleration) trajectories in classical physics. Thus, he argues, that a particle follows such a trajectory does not need a causal account in terms of forces or 'spacetime friction' - inertial motion is not caused. Nerlich's geometrical explanation relies on an identification: inertial motion is motion along a straight trajectory. The same explanation can be extrapolated to special and general relativity: default force-free trajectories are geodesic trajectories. Although this view is certainly much more nuanced than Brown's construal, it is still open to criticism from Brown's point of view. Nerlich's proposal assumes spacetime substantivalism, for he argues that the identification on which the geometric explanation relies is possible only by assuming that spacetime is real: "to parody Quine-no identities without entities. Only a realist can tell this story" (Nerlich 2010, 186). Now, if spacetime with a particular structure stands in and by itself, one may wonder why the dynamics that governs the behavior of physical systems corresponds to such a structure (see sections 3.1 and 4.1 below).
} 
complete explanatory picture demands a dynamical account of the explanans in terms of a fundamental quantum theory, a picture that is not (yet) available. However, the truncated interpretation can leave aside, in the meantime, the detailed description of how quantum microstructure of matter grounds Lorentz invariance. The truncated view, Brown argues, is enough to make the point that the arrow of explanation must go from Lorentz invariance to Minkowski structure:

One can postpone (as Einstein did) the detailed investigation into the forces and structures actually responsible for the phenomena that are paradigmatic of space-time's Minkowski geometry without thereby relinquishing the idea that these forces are, indeed, "actually responsible" for the phenomena in question and, hence [...], for spacetime having the structure that it has. (Brown \& Pooley 2006, 80)

What is important is that particular laws - a specific quantum field theory - could be solved and the solutions shown to have the requisite geometrical properties. (ibid, 81-82; cf. Brown \& Pooley 2001, section $11.3)^{3}$

\subsection{JANSSEN'S INTERPRETATION}

Now we turn to Michel Janssen's corner. He claims that the arrow of explanation points in the opposite direction: Minkowski spacetime structure explains Lorentz invariance. However, Janssen's view is not the stance that Brown rejects by means of the two arguments reviewed. He does not claim that physical objects have spacetime feelers that tell them how to move, nor does he claim that spacetime causally determines the dynamical behavior of physical systems. Rather, Janssen's view exploits the fact that paradigmatic relativistic phenomena are accounted for without reference to the microstructural constitution of matter. He states that such phenomena are kinematically grounded:

I claim that Minkowski spacetime explains Lorentz invariance. For this to be a causal explanation, Minkowski spacetime would have to be a substance with causal efficacy. Like Brown, I reject this view [...]. Minkowski spacetime explains by identifying the kinematical nature (rather than the cause) of the relevant phenomena. (Janssen 2009, 28)

Janssen (2009) illustrates his point by means of a conceptual and technical analysis of several special relativistic phenomena-optical effects associated to the Fresnel coefficient, the velocity dependence of mass, and the balanced torques of capacitors in the Trouton-Noble experiment. He concludes that they are all kinematical both in the broad sense of being independent of dynamical details, and in the narrow sense of being a manifestation of standard spatiotemporal behavior of physical systems. Janssen generalizes this conclusion by claiming that all paradigmatic special relativistic effects - all the effects which are a manifestation of Lorentz invariance-are kinematically grounded, both in the broad and in the narrow sense.

Now, it is the narrow sense that grounds Janssen's standpoint in the debate we are addressing. He states that the standard spatiotemporal behavior of physical systems is encoded in Minkowski structure. Thus, since paradigmatic relativistic effects are expressions of standard spatiotemporal behavior, Minkowski structure explains Lorentz invariance:

\footnotetext{
${ }^{3}$ That there is no available constructive explanation of the Lorentz invariance of all physical laws in terms of a fundamental quantum theory does not mean that special relativity does not provide an explanation of dynamical effects related to phenomena like length contraction. For example, special relativity explains why, as a consequence of length contraction, forces arise such that a rigidly rotating disk eventually explodes, and that a rope connecting two spaceships with equal accelerating programs eventually breaks (see Mermin 2005, chapter 13; Miller 2010). However, these explanations are chronogeometric, in the sense that they are grounded on Minkowski spatiotemporal structure (and, a fortiori, on Lorentz invariance, see section 5), but they do not constitute constructive explanations of Lorentz invariance. Such an explanation would require Lorentz invariance to result from fundamental quantum laws.
} 
Length contraction, time dilation, and the velocity dependence of mass are also kinematical in the narrow sense of exemplifying standard spatio-temporal behavior in special relativity. The Lorentz invariance that can be derived from the postulates (in conjunction with the assumption that time and space are homogeneous and isotropic) finds its natural interpretation in terms of the geometry of Minkowski space-time. (Janssen 2009, 39)

[Minkowski spacetime] explains all of them [special relativistic effects] by showing they need no explanation. Or, to put it less paradoxically, the statement that space-time is Minkowskian explains all of them in one fell swoop [...]. It commits one to assigning all manifestations of Lorentz invariance to the class of kinematical phenomena [...]. It tells scientists that there is nothing more to be learned from the study of specific elements in the class of kinematical phenomena. (ibid, 49)

Furthermore, Janssen states that, just as in the ether theory, in Brown's interpretation the fact that all the laws of physics are Lorentz invariance is left unexplained and constitutes a cosmic coincidence (Janssen 2002a, 2002b, 2009). On the contrary, in his proposal, that the laws of physics are Lorentz invariant gets explained in a unified and universal way by Minkowski spacetime structure. Janssen thus affirms that his interpretation is superior to Brown's because it involves a common origin inference:

Both in Lorentz's theory and in Brown's alternative interpretation of special relativity [...], Lorentz invariance is posited separately for different dynamical laws. [...] In Lorentz's theory as well as in Brown's proposal, it is a brute fact that these different laws are Lorentz invariant. In the orthodox version of special relativity this cosmic coincidence is traced to a common origin, Minkowski space-time. $(2009,48)$

Janssen's statement that the cosmic coincidence problem affects Brown's interpretation is too quick. If one considers that this proposal is, in the current state of science, truncated and provisional in the sense explained above, it is clear that a complete explanation of Lorentz invariance in terms of a fundamental quantum theory would provide for a unified explanation that all the laws of physics have this property ${ }^{4}$.

\section{TWO CRUCIAL ASSUMPTIONS}

Now that the essential points of the debate have been addressed, I will deal with two explicit assumptions shared by both corners in the dispute. As I will show, a critical assessment of these assumptions is crucial for a nuanced evaluation of the debate as a whole. In this section, I will unfold them and expose their justifications. In section 4, I will show that both assumptions are rather problematic.

\subsection{No SPACETIME ONTOLOGY}

Both parties agree in that the dispute is independent of specific presuppositions concerning the ontology of spacetime. This is a natural setup for the debate. Since the disputed subject concerns the interpretation of the foundations of a physical theory, it is a healthy attitude to leave aside ontological controversies that transcend the theory at stake. Thus, given that both sides in the discussion seem to be

\footnotetext{
${ }^{4}$ As I mentioned above, overlooking the truncated and provisional character of Brown's interpretation results in misleading judgments. Frisch (2011) is another example. In his construal of Brown's view he states that "according to Brown, it is a brute fact, which itself requires no further explanation, that the laws are Lorentz-invariant and it is this fact, which explains that Minkowski spacetime is the proper arena to represent non-gravitational phenomena" (176, my emphasis). In agreement with this (misconstrued) view, Frisch states that Janssen is mistaken when he looks for an explanation of Lorentz invariance. Although there are places where Brown states that Lorentz invariance is an unexplained brute fact in his interpretation (e.g., Brown \& Pooley 2006, 84), it is clear that in those passages he refers to the truncated, provisional version.
} 
quite close to a specific stance about the ontology of spacetime, both Janssen and Brown dedicate some words to try to disentangle their interpretations from commitments of this sort.

Janssen's proposal, at first sight at least, looks rather akin to a substantivalist stance. Let us recall that the kernel of his interpretation consists in that Minkowski spacetime constitutes the explanatory soil for Lorentz invariance of physical laws. Furthermore, he claims that this view involves a common origin inference: laws of physics are Lorentz invariant because such an invariance is an expression of default spatiotemporal behavior of physical systems, and, in turn, this standard spatiotemporal behavior is encoded in Minkowski structure. This may give the impression that Janssen is asserting that Minkowski spacetime is the entity that explains Lorentz invariance.

However, Janssen is fully explicit in that he does not presume a certain ontology of spacetime: "Special relativity as a physical theory is agnostic about the ontology of space-time. [...] Given that my argument is [...] not about ontology, it had better be independent of whether one is a relationist or a substantivalist about Minkowski space-time" $(2009,28)$. He denies a substantivalist commitment, for otherwise his interpretation would come close to the naive interpretation that Brown compellingly criticizes. According to Janssen, the way in which Minkowski structure explains Lorentz invariance is not causalMinkowski spacetime is not an entity whose ruts and groves determine the path of inertial objects. His proposal is, once again, that Minkowski structure is nothing but the formal codification of standard spatiotemporal behavior:

\footnotetext{
So in my view Minkowski space-time explains Lorentz invariance. For Brown and Pooley, however, Minkowski space-time is a "glorious non-entity" that can do no explanatory work. I agree that Minkowski space-time is not a substance with causal efficacy, so the sense of explanation I invoke is certainly not causal. I adopted the view, similar to Brown's, that Minkowski space-time encodes the default spatiotemporal behavior of all physical systems in a world in accordance with the laws of special relativity. (Janssen 2009, 49)
}

On the other hand, Brown's view, at least at face value, seems committed to a relationist conception of Minkowski spacetime - the title of the 2006 paper by Brown \& Pooley is "Minkowski Space-time: a glorious non-entity". Besides, to state that the appropriate spatiotemporal structure postulated by special relativity is Minkowskian because fundamental quantum laws are Lorentz invariant sounds rather close to an eliminative reduction of spacetime. However, Brown seems to be as aware as Janssen that overtly committing his interpretation to a specific position concerning spacetime ontology is a questionable maneuver. In the preface of his Physical Relativity, he warns us that

my book is not designed to be a defence of a Leibnizian/Machian relational view of space-time [...]. Although I have sympathies for this view, in my opinion the dynamical version of relativity theory is a separate issue and can be justified on much wider grounds, having essentially to do with good conceptual house-keeping. (2005a, ix)

Brown explicitly argues that his interpretation is the right stance even if we assume a substantivalist position. The reason is that since physical objects do not have 'spacetime feelers', they cannot be conceived as spacetime surveyors unless the dynamics that governs them corresponds to the self-standing spatiotemporal structure-this is the 'good conceptual house-keeping' Brown refers to in the quoted passage. In other words, if the laws that govern physical systems were not Lorentz invariant, then the chronogeometric structure of (self-standing) Minkowski spacetime could not be reflected at an operational level-rods and clocks could not survey it. Thus, for Brown, even in a substantivalist context, the Lorentz invariance of dynamical laws explains the Minkowski structure of spacetime: 
What needs to be stressed is that this conclusion [the dynamical interpretation] is appropriate not only for those who adopt an eliminative relationist stance towards the ontology of space-time [...]. Even when one's ontology includes substantival space-time structure, the symmetries of the laws governing material systems are still crucial in such structure gaining operational chronogeometric significance. (Brown \& Pooley 2006, 86; see also Brown \& Pooley 2001, section 11.6) $)^{5}$

\title{
3.2. CONSTRUCTIVE EXPLANATION
}

Now we can turn to the second crucial assumption. Janssen and Brown coincide in that the debate about the direction of the arrow of explanation that connects Minkowski structure and Lorentz invariance concerns a constructive explanatory arrow, in the sense of Einstein's 1919 distinction between theories of principle and constructive theories. Janssen and Balashov provide a characterization of this distinction that Brown \& Pooley $(2006,73)$ openly approve:

\begin{abstract}
In a theory of principle, one starts from some general, well-confirmed empirical regularities that are raised to the status of postulates (e.g., the impossibility of perpetual motion of the first and second kind, which became the first and second laws of thermodynamics). With such a theory, one explains the phenomena by showing that they necessarily occur in a world in accordance with the postulates. Whereas theories of principle are about the phenomena, constructive theories aim to get at the underlying reality. In a constructive theory one presupposes (a set) of model(s) for some part of physical reality (e.g., the kinetic theory modeling a gas as a swarm of tiny billiard balls bouncing around in a box). One explains the phenomena by showing that the theory provides a model that gives an empirically adequate description of the salient features of reality. (Balashov \& Janssen 2003, 331)
\end{abstract}

The reason why both Janssen and Brown demand that the arrow of explanation at issue must be constructive is that they agree in that constructive explanations are essentially superior to explanations of principle. An expression of this judgment can be extracted from Balashov \& Janssen's description of the distinction. Whereas theories of principle superficially explain (or merely describe) the phenomena by referring to abstract and general nomological principles that act as constraints, constructive theories tell us why the phenomena occur by unraveling the reality behind the veil of appearances. In other words, only constructive explanations open the black box. In this sense, Janssen states that "principle theories are not explanatory. Explanations are about the reality behind the phenomena (be it about their causes or about their nature). Principle theories [...] are agnostic about that" $(2009,38)$. Thus, he states that physical phenomena that are a manifestation of Lorentz invariance are constructively explained by Minkowski spacetime:

Minkowski (1909) did for special relativity, understood strictly as a principle theory, what Boltzmann had done for the second law of thermodynamics. It turned special relativity into a constructive theory by

\footnotetext{
${ }^{5}$ Butterfield (2007) values this argument as an important moral that can be drawn from Brown's view: “The point here is not just that it would be wrong to infer from a quantity being called a metric that it mathematically represents (what the theory predicts about) the readings of rods, and-or clocks and-or other instruments for measuring lengths and time-intervals. That is obvious enough: after all, a quantity might be given an undeserved, even tendentious, name. But also, we should not infer from the fact that in the theoretical context, the quantity is mathematically appropriate for representing such behavior, that it does so. For example, in the Gauss-Riemann conception of length as given by line-integrals of $d s=$ $\sqrt{\left(g_{i j} d x^{i} d x^{j}\right)}$, the symmetric tensor $g_{i j}$ is appropriate. More specifically, for relativity's spatiotemporal lengths, $g_{i j}$ is to have Lorentzian signature. But such a tensor might well not represent measured lengths or times. After all, a theory might contain two such tensors, just one of which represents such matters" (308-309). In other words, theoretical terms earn their metric meaning on the basis of the specific dynamics of ideal rods and clocks (cf. Hoving 2013, 26). This moral will be important below. Using it, in section $4.1 \mathrm{I}$ will argue against Brown's statement that his interpretation can be adopted in a substantivalist context.
} 
providing the concrete model for the reality behind the phenomena covered by the principle theory. (Janssen 2009, 40)

Brown's view assumes a similar stance regarding the principle-constructive dichotomy - a stance that refers to Einstein's own evaluation of the distinction. To illustrate, he invites us to consider the explanation of principle that thermodynamics offers for the efficiency limits of a single-piston engine. These limits are given by a ratio between the temperatures of the two heat reservoirs, a ratio that is in turn determined by the principle of the impossibility of a perpetual motion machine of the second kind. Brown asserts that, simple and straightforward as it is, this explanation loses out, in terms of physical insight, to the constructive explanation provided by statistical mechanics. Although he is not explicit about it, it is likely that Brown assigns more physical insight to constructive theories than to theories of principle inasmuch as the former carry an ontological and causal baggage that is absent in the latterin this sense, constructive explanations tend to be mechanical. In agreement with Einstein, Brown concludes that

Principle theories are typically employed when constructive theories are either unavailable, too difficult to build, or relatively unwieldy. For according to Einstein, 'when we say that we have succeeded in understanding a group of natural phenomena, we invariably mean that a constructive theory has been found which covers the processes in question'. (Brown 2005b, S86)

Brown's interpretation thus involves an attempt to fulfill an explanatory gap that special relativity, understood as a theory of principle, leaves empty. In this sense, his stance concerning the direction of the arrow of explanation between Lorentz invariance and Minkowski structure, by definition, requires it to be a constructive arrow. To state that the structure of spacetime is Minkowskian because fundamental dynamical laws are Lorentz invariant certainly involves a constructive explanation-for Brown, the Lorentz invariance of dynamical (quantum) laws is an expression of the way in which the ultimate blocks of nature are made. Although a complete constructive explanatory picture of Lorentz invariance is not (yet) provided by a fundamental quantum theory, the truncated version of his standpoint is an attempt to ease the dissatisfaction that many voices expressed with respect to the way in which special relativity explains (cf. Brown 2005a, chapter 7) ${ }^{6}$.

\section{DECONSTRUCTING THE DEBATE}

Harvey Brown asserts that special relativity must be interpreted in such a way that Lorentz invariance constructively explains Minkowski spacetime structure, and he states that this view does not require a relationist eliminative spacetime ontology. Michel Janssen states that Minkowski spacetime structure, understood as encoding standard spatiotemporal behavior of physical systems, constructively explains that physical laws are Lorentz invariant, and he claims that this view does not presuppose a substantivalist stance. I will now undertake a critical evaluation of these opposing outlooks. First, I will show that, despite their remarks to the contrary, Brown's view indeed presupposes spacetime

\footnotetext{
${ }^{6}$ A particularly clear expression of this dissatisfaction is given by an often-quoted passage from Pauli's seminal book on special relativity: "Should one then completely abandon any attempt to explain the Lorentz contraction atomistically? We think that the answer to this question should be No. The contraction of a measuring rod is not an elementary but a very complicated process. It would not take place except for the covariance with respect to the Lorentz group of the basic equations of electron theory, as well as of those laws, as yet unknown to us, which determine the cohesion of the electron itself" (Pauli 1921, 15).
} 
relationism, and that Janssen's certainly presupposes substantivalism. Furthermore, I will argue that the demand to interpret special relativity in constructive terms - shared by both parties -is unjustified.

\subsection{SURREPTITIOUS SUBSTANTIVALISM, SURREPTITIOUS RELATIONISM}

Janssen's statement that his interpretation is not committed to spacetime substantivalism is deeply problematic. The problem results from his demand for a constructive arrow of explanation. According to Janssen's own rendition of Einstein's distinction, constructive theories explain by unraveling the reality behind the phenomena. Now, if spacetime is not conceived as a self-standing entity, but as the formal encoding of spatiotemporal behavior, how can it be the constructive explanation of anything? The very definition of a constructive explanation requires that if spacetime is to be assigned a constructive explanatory role, then it must be hypostatized. If we accept Janssen's view that Minkowski spacetime structure encodes the standard spatiotemporal behavior of physical systems, it cannot be the constructive explanation of Lorentz invariance unless it is regarded as the reality that somehow determines such a behavior.

We can level the same criticism by referring to Janssen's common origin inference argument. If the common origin is to constructively explain the Lorentz invariance of physical laws, it is natural to assume that we are talking of a physically real origin. If Minkowski structure is nothing but the formalconceptual encoding of the standard spatiotemporal behavior of physical systems, then how is it supposed to be the common origin that constructively explains Lorentz invariance? In short, if spacetime is not hypostatized, at most it can provide a formal-unificationist explanation of Lorentz invariance. Janssen tries to make room for his interpretation of special relativity in the definition of constructive explanations when he states that "explanations are about the reality behind the phenomena (be it about their causes or about their nature)" (2009, 38; my emphasis). However, to extend the notion of a constructive explanation by means of the rather vague statement that they can refer to the nature of the phenomena boils down to blurring Einstein's distinction.

It may be objected that Janssen's view can be taken as claiming that spacetime as a structure rather than as an entity plays the constructive explanatory role with respect to Lorentz invariance. Moreover, the spatiotemporal structure at issue may be characterized as an ultimate and fundamental structure of relations that is not reducible or eliminable by reference to dynamical laws and constituents of matter. If we understand Janssen as stating that Minkowski spacetime, considered as a structure of relations of this kind, explains Lorentz invariance in a constructive way, then one could think that his interpretation can be formulated in a relationist framework after all.

However, this construal of Janssen's view is equally committed to a hypostasis of spacetime. To see why, we should be aware that the term 'substantivalism' is a misnomer. In a broad ontological sense, the substantivalist thesis states that spacetime exists over and above spatiotemporal objects - it is not ontologically reducible to (relations between) them. The relationist view denies such a thesis, it asserts that physical objects are the ultimate and primary constituents of the world, and that spacetime is nothing but the expression of a certain type of relations between them-relationism regards spacetime as a class of relations that are instantiated in physical objects, and it denies its independent existence. Now, the substantivalist thesis can take many forms, depending on which specific ontological description it assigns to spacetime (e.g., manifold substantivalism, metric field substantivalism, spacetime structuralism), but all those variants agree in that spacetime is ontologically independent with respect to physical objects, they all state that it exists in and by itself. This is the reason why the term 'substantivalism' is confusing. It would be better to use the term 'absolutism' for this basic thesis, so that 'substantivalism' would refer to a particular form of the absolutist thesis. 
Now I can introduce my criticism of Janssen's interpretation in more clear terms. To state that Minkowski spacetime constructively explains Lorentz invariance necessarily assumes-given the definition of constructive explanations - the absolutist thesis. This is so even if Minkowski spacetime is conceived as an irreducible structure of relations. To further elucidate my point, let us consider two characterizations of 'spacetime structuralism', a recent proposal that describes spacetime as a system of irreducible relations:

This option, which I call structural realism, sides with the later doctrine [relationism] in defending the relational nature of spacetime, but argues with the former [substantivalism] that spacetime exists, at least in part, independently of particular objects and events. (Dorato 2000, 1607-1608).

Dorato's formulation of spacetime structuralism as a thesis that conceives spacetime as a class of irreducible relations makes it apparent that, just as substantivalism, it is a particular form of the absolutist thesis. In Janssen's interpretation, if spacetime is to be understood as a structure of relations, and, at the same, as the constructive explanation of Lorentz invariance, then spacetime structuralism as an instance of spacetime absolutism must be assumed. If spacetime as a structure of relations were reducible or eliminable by reference to physical systems and dynamical laws, it could not play a constructively explanatory role. Consider also Slowik's characterization:

First, mathematical structuralism can be classified according to whether the structures are regarded as independent or dependent on their instantiation in systems (ante rem and in re structuralism, respectively), where a 'system' is loosely defined as a collection of 'objects' and their interrelationships. Ante rem structuralism [...] is thus closely akin to the traditional 'absolute' conception of spacetime, for a structure is held to 'exist independent of any systems that exemplify it' [...]. Yet, since 'system' (and 'object') must be given a broad reading, without any ontological assumptions associated with the basis of the proposed structure, it would seem that substantivalism would not fit ante rem structuralism, as well. The structure of substantivalism is a structure in a substance, namely, a substance called 'spacetime', such that this unique substance 'exemplifies' the structure (whereas ante rem structure exists in the Platonic sense as apart from any and all systems that exemplify it). The substantivalist might try to avoid this implication by declaring that their spacetime structures are actually closer in spirit to a pure absolutism, without the need of any underlying entity (substance) to house the structures (hence, 'substantivalism' is simply an unfortunate label). (Slowik 2005, 152)

Once again, if we take Janssen's interpretation as stating that Minkowski spacetime, as a structure of relations, constructively explains Lorentz invariance, then ante rem spacetime structuralism must be assumed. This is already enough to see that Janssen's view necessarily takes a side in the substantivalismrelationism debate (or, better, in the absolutism-relationism debate). He explicitly takes it that his proposal is not about spacetime ontology and that special relativity is silent about it: the theory does not tell us whether spacetime is ontologically dependent or independent. Thus, if Minkowski spacetime is a structure of relations, Janssen's argument should be able to make sense in the context of in re spacetime structuralism as well. But this is not possible, for if the relations are ontologically dependent on their instantiation on the relata, then the constructive explanatory work is not performed by Minkowski spacetime, but by the physical objects, and the dynamics that governs them, on which the Minkowski structure of relations is instantiated. In an ontologically relationist in re version of spacetime structuralism, the reality behind the phenomena is given by the physical objects and the corresponding dynamical laws, not by Minkowski structure.

On the other hand, Brown's dynamical interpretation of special relativity is certainly committed to a relationist eliminative stance. The reason is that if a substantivalist version of his argument is asserted, 
the result is an ontological fluke. Let us return to the three theses that feature Brown's criticism of the standard view:

i) Physical objects do not have spacetime feelers, so they cannot accommodate their behavior to its structure.

ii) As a matter of logic alone, the structure of a self-standing spacetime does not determine the symmetries of physical laws.

iii) If physical objects - ideal rods and clocks - are to survey the structure of spacetime; the symmetries of the dynamical laws that govern them must correspond to such a structure (notice that iii) connects to $i$ ) and ii)).

If we accept these appealing theses, the following counterparts then look very convincing as well:

$i^{\prime}$ ) A self-standing spacetime does not have object feelers, so it cannot adapt its structure to the symmetries of the dynamical laws.

ii') As a matter of logic alone, the symmetries of the dynamical laws do not fix the structure of spacetime (again, Lorentz's ether theory constitutes an instance of this thesis).

Let us now assume Brown's interpretation in a substantivalist (or absolutist) context. Consider once again his thesis iii), that if rods and clocks are to be surveyors of the structure of spacetime, the symmetries of the laws that govern them must correspond to its structure. Now, given $i^{\prime}$ ) and $i i^{\prime}$ ), the fact that the dynamical symmetries correspond to the structure of spacetime becomes a cosmic coincidence. Since the structure of a self-standing spacetime cannot adapt to the dynamical symmetries, nor can it be logically determined by them, spacetime could have a structure that does not reflect the dynamical symmetries of the laws. In this case, rods and clocks could not work as surveyors of spatiotemporal structure (as in Lorentz's theory), but in special relativity ideal rods and clocks do survey Minkowski spacetime, of course. Thus, a substantivalist that endorses the aforementioned theses in Brown's view gets committed to a cosmic coincidence between Minkowski spacetime structure and Lorentz invariance. Thus, in a substantivalist setup, Brown's view does not work, for Lorentz invariance cannot explain Minkowski structure - they have to be independently posited.

The reader may complain that if we consider general relativity into the picture, and if we assume a substantivalist position of the bare-manifold type, we could formulate Brown's interpretation in a substantivalist context. The idea is to conceive that spacetime, as a self-standing entity, has only topological properties, and that its metric structure is dynamically induced by the presence of mass-energy according to Einstein field equations. The key point in this response is that Minkowski spacetime describes a tangent spacetime in a global general relativistic spacetime.

However, Brown himself states (2005a, section 1.5; Brown \& Pooley 2001, section 11.6) that once the metric field is determined in a solution of the field equations, the operational significance of the local Minkowski metric does not yet obtain. The missing ingredients are the strong equivalence principle and the clock-hypothesis ${ }^{7}$ (along with its analogue for rods), without these requirements, rods and clocks cannot be spacetime surveyors. Now, the clock hypothesis and the strong equivalence principle are extra postulates that do not come included in solutions of the field equations. For example, though the Minkowski metric of a tangent spacetime is guaranteed, the dynamics governing non-gravitational

\footnotetext{
7 "that in 'small enough' regions of space-time, for most practical purposes the physics of the non-gravitational interactions takes its usual Lorentz covariant form", and "that when a clock is accelerating, the effect of motion on the rate of the clock is no more than that associated with its instantaneous velocity", respectively (Brown 2005a, 9).
} 
physical processes in it could have been non-Lorentz invariant (cf. footnote 4 above). In a substantivalist context, this means that the fact that the dynamics of ideal rods and clocks reflects the structure of a selfstanding spacetime is an unexplained coincidence, even if we consider the gravitational theory into the picture. Thus, notwithstanding the link between dynamics and metric in general relativity, the Lorentz invariance governing ideal rods and clocks does not induce the Minkowski metric of a (bare-manifold) self-standing spacetime. Hence, the correspondence between Lorentz invariance and Minkowski structure is still an ontological fluke: in a bare-manifold substantivalist context, Lorentz invariance cannot explain Minkowski structure ${ }^{8}$.

More generally, my point is that Brown's interpretation is committed to a form of relationism, for, in a substantivalist setup, his views concerning spatiotemporal metric and dynamics of rods and clocks lead to the aforementioned cosmic coincidence-so that Lorentz invariance is unable to explain Minkowski structure. He argues that spacetime cannot determine Lorentz invariance, neither causally nor logically. Now, if we concede this, and assume that Minkowski spacetime is a self-standing entity, it is not possible to conceive that Lorentz invariance constructively explains Minkowski structure-the latter must be independently postulated. In other words, the reasons that lead us to assert that the Minkowski structure of a self-standing spacetime cannot determine Lorentz invariance neither causally nor logically, also lead us to assert that Lorentz invariance cannot determine the structure of a self-standing spacetime, neither causally nor logically9.

The upshot of this section is that, despite their remarks to the contrary, Janssen's and Brown's proposals are indeed associated to a position in the controversy between substantivalists (absolutists) and relationists. When an interpretive thesis concerning the foundations of a physical theory is defended, it is a healthy attitude to avoid getting involved in philosophical issues that transcend the theory addressed. However, given the nature of the arguments posited by our authors, it is impossible for them to remain neutral about the philosophical issue of the ontology of spacetime. Both Janssen and Brown must take sides in the relationism-substantivalism controversy in their interpretations of the foundations of special relativity - a physical theory which, in and by itself, is agnostic about that controversy.

\subsection{A CONSTRUCTIVE ARROW?}

Now we turn to a critical appraisal of the demand that the arrow of explanation at issue has to be constructive. For both authors, the justification of this insistence relies on an evaluation of Einstein's distinction in which constructive explanations are deemed essentially superior to explanations of principle. Given this assumed hierarchy between the types of explanations that Einstein distinguishes, Janssen and Brown state that there is an urgent need to provide a constructive version of the explanatory

\footnotetext{
${ }^{8}$ In Brown \& Pooley's own words: "it is mysterious to us how the existence of a local approximate Minkowski geometry entails the Lorentz covariance of the laws of the non-gravitational interactions. Theories postulating a Lorentzian metric but which violate minimal coupling would involve non-Lorentz covariant laws" $(2001,270)$. The problem for a substantivalist version of Brown's interpretation is that the converse also holds: the local approximate Minkowski geometry does not result from the Lorentz invariance of non-gravitational interactions, even though it follows from the Einstein field equations (as the metric of a tangent spacetime).

${ }^{9}$ John Norton (2008) introduced a criticism that seems to be the total opposite. He argues that Brown's dynamical project can be successful only if it assumes spacetime substantivalism. However, he picks on a wholly different issue. Norton points out an alleged technical shortcoming in Brown's interpretation, namely, that spacetime geometry cannot be formally obtained solely from the properties of matter-recourse to spatiotemporal presumptions is unavoidable (cf. Hagar \& Hemmo 2013). An important aspect in Norton's argument is that "the key to the reconstruction [of spacetime structure] is the Lorentz covariance of all matter theories. It is a 'brute fact' according to Brown" (Norton 2008, 825). Once again, overlooking the truncated and provisional aspect of Brown's proposal results in a misleading evaluation: if Lorentz invariance results from a fundamental quantum theory, it would not be the ultimate ground for the reconstruction, so the objections posited by Norton could be, at least in principle, avoided or overcome. A full (non-truncated) version of Brown's interpretation is not necessarily problematic in Norton's sense.
} 
foundations of special relativity. Accordingly, they both introduce their positions in the debate in the form of a constructive arrow of explanation (pointing in opposite directions) between Minkowski spacetime and Lorentz invariance. Now I will show that even assuming that the hierarchy they adopt between constructive explanations and explanations of principle is adequate, it does not follow that there is an urgent demand for a constructive version of the explanatory foundations of special relativity.

The hierarchical evaluation at issue can be characterized by means of the following principle

$P_{1}$ ) In general, given a realm of phenomena $E$ for which there is a theory of principle $T_{p}$ and a corresponding constructive theory $T_{c}, T_{c}$ is explanatorily more fundamental than $T_{p}$ with respect to $E$.

This is a plausible principle. Its rationale seems to be something like the following: if a constructive theory explains a realm of phenomena for which there is also a theory of principle, it means that there is a black box enclosing the reality behind the phenomena that the constructive theory, but not the theory of principle, opens. This is a common characterization of the case of thermodynamics and statistical mechanics, for example. However, in spite of its prima facie plausibility, the principle is controversial. $P_{1}$ seems to rely on the view that when there is a constructive theory $T_{c}$ and also a theory of principle $T_{p}$ for the same realm of phenomena $E$, then $T_{p}$ can be reduced (ontologically and/or epistemologically) to $T_{c}$. Now, the corresponding reduction in the paradigmatic case of statistical mechanics and thermodynamics is far from being settled, and the problems that such a reduction must face (if it is possible at all) are deep and difficult. Furthermore, the very concept of intertheoretic reduction is far from clear, and there are several competing models of reduction (see Sklar 1995, chapter 9). These problems can be taken as casting doubts on $P_{1}$.

Anyhow, for the sake of the argument, let us assume that $P_{1}$ is a sound principle. Even under the assumption that constructive explanations are essentially superior to explanations of principle, the demand for a constructive version of special relativity that Janssen and Brown share is unjustified. This exigency relies not only on $P_{1}$, but also on the view that if special relativity cannot be turned into a constructive theory, then it is explanatorily deficient and poor-recall Janssen's remark that theories of principle are not explanatory, and Brown's statement that they are only useful as a provisional surrogate when a constructive theory is not available. The demand for a constructive arrow of explanation goes further than the comparative evaluation between explanations of principle and constructive explanations expressed in $P_{1}$. This principle states that a constructive explanation is more fundamental than an explanation of principle when they are both available for the same realm of phenomena. However, the thesis that if we have an explanation of principle for a certain realm of phenomena, then we are in a wanting situation unless a constructive explanation is also at hand, is a stance that also commits to a further principle

$P_{2}$ ) For every realm of phenomena $E$ for which there is a theory of principle $T_{p}$, there is a constructive theory $T_{c}$.

Principle $P_{1}$, in and by itself, is not enough to sanction the urgent demand for a constructive interpretation of special relativity that Janssen and Brown share. We may assume that when we have a theory of principle and a constructive theory for the same realm of phenomena, the latter is explanatorily more fundamental than the former. However, from this assumption it does not follow that if we have only a theory of principle $T_{p}$ for a certain realm of phenomena $E, T_{p}$ is explanatorily incomplete, deficient and poor, for, after all, $T_{p}$ may be the ultimate explanation for $E$. In this case, $T_{p}$ would explain everything 
there is to explain. Thus, the view that special relativity is an explanatorily deficient theory that needs to be fixed or completed by means of a constructive version crucially relies on $P_{2}$.

Now, and this is the crucial point, $P_{2}$ has a very different status than $P_{1}$. The appeal and plausibility of $P_{1}$ can be assessed in terms of the very definitions of the kinds of theories and explanations involved (leaving aside, for the sake of the argument, the problems with the concept of reduction mentioned above). $P_{2}$, on the contrary, is a metaphysical principle. It is clear that we cannot know whether $P_{2}$ is true or not. That we do not have a constructive theory for a realm for phenomena for which there is a theory of principle is not a refutation of the principle, for it may just be a reflection of our incapacity to formulate it. On the other hand, if for all actual theories of principle there were a corresponding constructive theory, that could not count as a confirmation of $P_{2}$. Since it is a universal principle that refers to contingent cases, we cannot characterize it as empirically vindicated, no matter how strong its inductive support may be. Thus, in terms of its truth, $P_{2}$ is highly problematic, if decidable at all. In turn, the absolute demand for a constructive interpretation of special relativity is as questionable as the truth of $P_{2}$-there just may not be a constructive counterpart for special relativity as a theory of principle ${ }^{10}$. Furthermore, as we will see, Einstein's theory is explanatorily powerful and satisfactory as it is. There is no need to repair it in explanatory terms.

\section{TWO SIDES OF THE SAME COIN}

I will now argue that a more nuanced, adequate and fruitful construal of the explanatory foundations of special relativity is that Lorentz invariance and Minkowski structure do not constitute two features of the theory such that one has to be explained by the other. Rather, they can be understood as two sides of a single coin, so there is no need to demand for an arrow of explanation connecting them. I will also argue that the fact that Minkowski structure and Lorentz invariance are connected in an analytic way actually clarifies the source of the explanatory power of the theory.

In order to unfold my first thesis, let us take a quick look at the historical sequence of events concerning the formulation of special relativity. In the "kinematical part" of his first paper on the theory, Einstein (1905), departing from the relativity principle and the light principle, along with the assumption of the homogeneity of space and time, derived a set of coordinate transformations ${ }^{11}$. He immediately recognized that these transformations involved revolutionary physics in the sense that they implied a rejection of some basic elements in the conceptions of space and time underlying Newtonian mechanics - most notably, the rejection of absolute simultaneity. Einstein readily noticed that his theory rendered lengths and temporal durations frame-relative. Furthermore, in the "electrodynamical part" of the paper, Einstein showed that the coordinate transformations he obtained predicted and explained novel phenomena, such as the relativistic Doppler effect and the velocity dependence of mass. Thus, we can safely state that in his 1905 paper, Einstein provided an explanation of principle of the coordinate transformations (and, a fortiori, of Lorentz invariance) - transformations which in turn explain the mentioned relativistic effects.

\footnotetext{
${ }^{10}$ As it will clear below, if there is no constructive version or counterpart for special relativity as a theory of principle, it does not follow that the theory cannot be explanatorily extended or enriched in some other way. There is no reason at all to understand Einstein's distinction between explanations of principle and constructive explanations as an exhaustive dichotomy.

${ }^{11}$ Contemporary studies in the foundations of special relativity have unfolded further subtle assumptions that need to be taken on board in order to derive the Lorentz transformations in their precise form. See, for example, (Friedman 1983, chapter IV, section 2).
} 
Shortly after, Hermann Minkowski (1909) showed that the coordinate transformations that Einstein derived in the 1905 paper are the symmetries of a four-dimensional manifold whose metric structure is given by the invariant expression $\sqrt{c^{2} d t^{2}-d x^{2}-d y^{2}-d z^{2}}$. That is, we can safely state, without getting involved in interpretive controversies, that Minkowski completely and precisely unfolded the spatiotemporal structure contained in Einstein's theory. In other words, the German mathematician showed that the symmetries of physical laws that Einstein obtained in 1905 from the two principles, are also the symmetries of what we nowadays call Minkowski spacetime.

Coming back to our debate, Janssen states that Minkowski's achievement constitutes a constructive explanation of Einstein's 1905 breakthrough. He argues that the Lorentz coordinate transformations are the appropriate symmetries of the laws because of the spacetime structure that the theory postulates. Brown, on the contrary, argues that we must understand special relativity in such a way that the physical foundations of Minkowski spacetime are given by dynamical properties of matter, properties that are expressed in the Lorentz invariance of physical laws. The thesis that I would like to introduce here is that both these views constitute overinterpretations of the connection between Lorentz invariance and Minkowski spatiotemporal structure. We do not need an arrow of explanation like the one that Brown and Janssen look for in order to make sense of the explanatory foundations of special relativity. As I explained in the simple and uncontroversial historical sketch above, what Minkowski did was not to provide for the physical grounds of the results of Einstein's 1905 paper. Rather, Minkowski's contribution is a conceptual display of Einstein's work, in the sense of an overt description of the spatiotemporal structure underlying the theory - a structure that Einstein had already glimpsed.

Let me introduce two important points of clarification. First, this minimal reading of the significance of Minkowski's contribution is neither committed to a substantivalist (absolutist) nor to a relationist conception of spacetime. Minkowski spacetime is the conceptual chronogeometric scaffolding underlying Einstein's findings. Second, this account of the link between Lorentz invariance and Minkowski structure can be grasped by the term 'analytic'. Kant defined analytic propositions as those in which the predicate concept is contained in the subject concept. In this sense, analytic sentences are explicative rather than explanatory - they display or define the meaning of the subject concept, but they do not explain it (unless one assumes too broad a notion of explanation). In simple words, what I propose here is that Lorentz invariance (under the assumption that rods and clocks are reliable chronogeometric surveyors ${ }^{12}$ ) and Minkowski spacetime structure are like the subject and the predicate of an analytic proposition. Furthermore, our subject and predicate can shift roles and explicate each other, so the analytic connection we are dealing with is also definitional. This is why any attempt to provide a physical explanation of one in terms of the other turns into a circle: why is spacetime Minkowskian? Because the laws of physics are Lorentz invariant. Why are the laws of physics Lorentz invariant? Because spacetime is Minkowskian. Thus, rather than a unidirectional explanatory arrow, what connects Lorentz invariance and Minkowski spacetime structure is a bidirectional explicatory arrow ${ }^{13}$.

\footnotetext{
12 This proviso, which is at the heart of relativity theory, is crucial. Without it, the analytic connection would not hold, as in Lorentz's ether theory.

${ }^{13}$ The bidirectional-explicative connection between Lorentz invariance and Minkowski spacetime becomes quite clear when one considers that Minkowski structure can be obtained from the Lorentz transformations, and vice versa. The coordinate transformations entail that the quantity $\sqrt{c^{2} t^{2}-x^{2}-y^{2}-z^{2}}$ is invariant, and Minkowski structure can be obtained by considering such a quantity as the spacetime metric. On the other hand, in order to obtain the Lorentz transformations from Minkowski spacetime "we start by postulating the geometrical structure of Minkowski space-time. We then look for the group of transformations of Minkowski space-time onto itself that will preserve that geometrical structure: this turns out to be the Lorentz group. Finally, using the geometrical structure of Minkowski space-time, we define the class of inertial frames and show that any two inertial frames are related by a Lorentz transformation" (Friedman 1983, 138-139).
} 


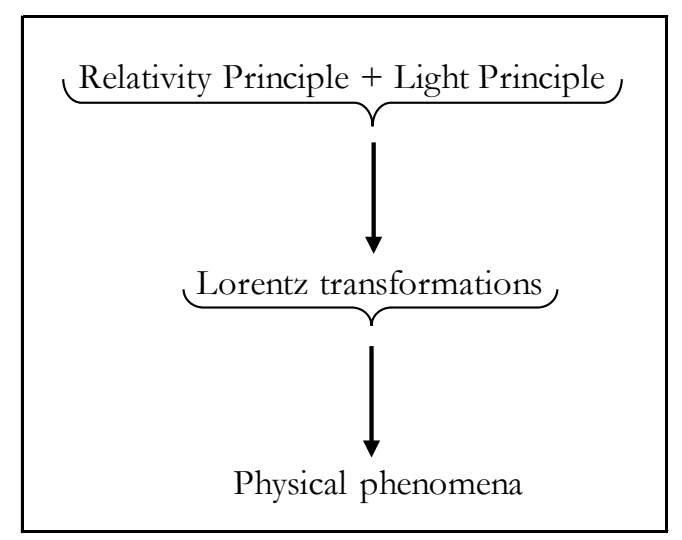

Figure 1

Although my proposal denies that there is an explanatory relation between Minkowski spacetime and Lorentz invariance, I do not characterize Minkowski's contribution as explanatorily empty. Fig. 1 represents the explanatory structure of Einstein's work. The top-down arrows indicate that, in its 1905 version, special relativity is a theory that corresponds quite neatly to Einstein's characterization of a theory of principle. The light principle and the relativity principle are the constraining principles that explain the phenomena via the Lorentz transformations. Thus, the theory, in its 1905 version, offers an explanation of principle as an account of Lorentz invariance, for the coordinate transformations are derived from the two principles (plus suitable assumptions).

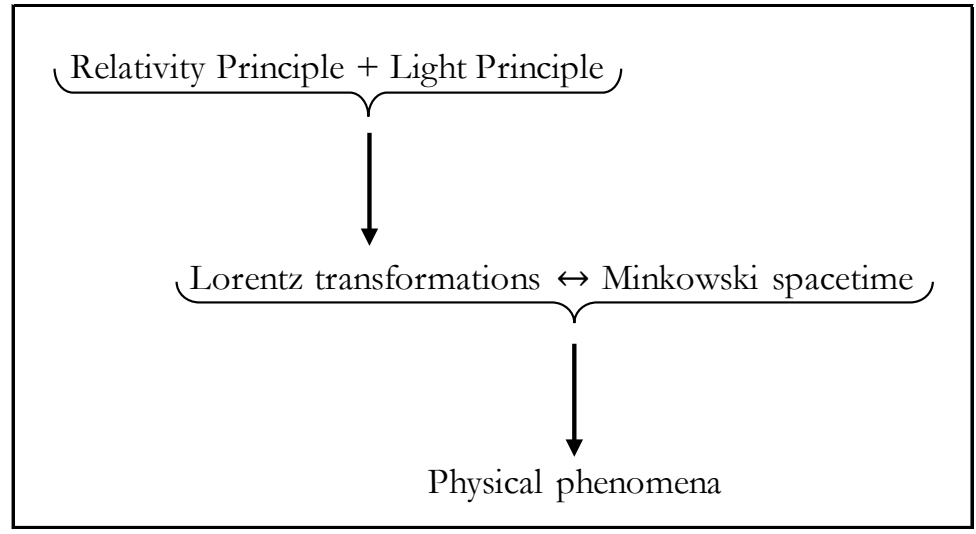

Figure 2

Fig. 2 describes the explanatory structure of special relativity after Minkowski's work. The horizontal bidirectional arrow connecting the Lorentz transformations and Minkowski spacetime structure is not explanatory. However, the figure intends to show that Minkowski spacetime structure is not explanatorily idle. Although it does not explain Lorentz invariance (nor is it explained by it), that Minkowski spacetime is the unfolding of the chronogeometric structure of the theory certainly constitutes a source of further intelligibility of physical phenomena, not of the Lorentz invariance of physical laws.

Let us consider for example the velocity dependence of mass. In 1905 Einstein showed that it is a phenomenon that is predicted and explained by the Lorentz transformations, in the sense that it is a phenomenon that must occur in a world that obeys the two principles. After Minkowski's work, special relativity explains this phenomenon also by showing that it is essentially connected to the chronogeometric structure-understood as a conceptual scaffolding-that the theory postulates. More generally, 
Minkowski's contribution was to show that paradigmatic relativistic effects connected to Lorentz invariance are instances of default spatiotemporal behavior-as Janssen would put it. Thus, in order to appreciate the explanatory power that Minkowski's work made explicit in Einstein's theory, we need not interpret special relativity in such a way that there is an explanatory arrow between Lorentz invariance and spacetime structure. Rather, the explanatory power of the theory resides in an arrow that goes from [Lorentz invariance $\leftrightarrow$ Minkoswki structure] to physical phenomena.

To be clear, this outline of the explanatory nature of special relativity does not purport a constructive version of the theory. The arrow of explanation from [Lorentz invariance $\leftrightarrow$ Minkoswki structure] to physical phenomena is not constructive. Minkowski spacetime is considered as a conceptual scaffolding, not as the reality behind the phenomena. However, although Minkowski did not do for special relativity what Boltzmann did for thermodynamics, his work was indeed explanatorily novel. The way in which spacetime structure contributes to make physical phenomena intelligible does not amount to an explanation in terms of mere principles either (Einstein's distinction does not exhaust all possible types of explanations in physics), for Minkowski's work unfolded the chronogeometric account of physical phenomena that special relativity offers. In Einstein's 1905 paper the very concept of spacetime is completely absent. Thus, although he was able to notice that his two principles implied the rejection of Newtonian kinematics, he (and anyone else before Minkowski's conference) could not realize that his theory introduced a spacetime with a particular metric that jettisons Newtonian spacetime. Consequently, a chronogeometric explanation is not possible in the 1905 version of special relativity. The unfolding of the analytic connection between Einstein's new kinematics - Lorentz invariance-and Minkowski structure is thus explanatorily novel. Borrowing Janssen's expression once again, Minkowski made it clear that relativistic phenomena are manifestations of standard spatiotemporal behavior.

In order to further clarify the epistemic content of chronogeometric explanations in special relativity, we can refer to Robert Disalle's $(1994,1995,2006)$ theses on the history and foundations of spacetime theories. Disalle confronts the substantivalism-relationism debate by challenging the way in which both sides in the dispute conceive the connection between spatiotemporal structure and physical phenomena. Against the relationist, Disalle argues that spatiotemporal structure is not an abstraction based upon the relations observed between physical phenomena. Such a structure, he claims, comprises the conceptual framework that constitutes and makes those relations intelligible in the first place (1994, section $4 ; 1995,319-320)$. Against the substantivalist, Disalle states that spatiotemporal structure is not a causal explanation of the behavior of physical objects. That is, that chronogeometric structure is constitutive of observable spatiotemporal relations does not mean that spacetime is an underlying entity that causally-constructively accounts for the phenomena. Spatiotemporal structure in physical theorizing plays a strictly epistemic role:

Space-time theories do not claim that some unobservable thing is the cause of observable effects. Instead they make a more restricted, but perhaps more profound and certainly more useful claim: that particular physical processes, governed by established physical laws, can be represented by aspects of a geometrical structure in the universe. $(1995,333)$

In the particular case of special relativity, Disalle $(2006,114)$ coincides with my (I think, uncontroversial) view that Minkowski structure expresses the spatiotemporal framework that corresponds to physical laws whose symmetries are given by the Lorentz coordinate transformations. Minkowski structure and Lorentz invariance mirror each other, so there is no point in taking one as explanandum and the other as explanans. However, by displaying the spatiotemporal structure contained in Einstein's 1905 
work, Minkowski bestowed special relativity with some explanatory power that, in its original formulation, was not fully explicit:

Minkowski's account does have a deeper though perhaps less obvious significance. For it reveals just how simple and direct is the connection between the structures of space and time and the assumptions we make about physics. The claim at the heart of Minkowski's analysis is, at the same time, extremely far-reaching and extremely modest: it is the claim that a world in which special relativity is true, simply, is a world with a particular space-time structure. (ibid, 116-117)

In sum, special relativity does not require a constructive version in order to be satisfactorily and substantially explanatory. Einstein's 1905 original formulation is indeed explanatory. The two principles render physical phenomena intelligible exactly in the way in which theories of principle explain the world: by means of abstract and general constraints ${ }^{14}$. Besides, Minkowski's work made it explicit that special relativity is not only a theory of principle, but also a spacetime theory, thus deepening and extending its explanatory power in chronogeometric terms.

Yet, one could still argue that there is a gap that needs to be filled. One could demand, as Brown and Janssen do, for a constructive interpretation of the theory. However, as I explained above, this demand relies on an uncritical endorsement of $P_{2}$. The ultimate explanations of relativistic phenomena may be the principle and the chronogeometric ones that Einstein's theory offers, or an eventual quantum gravity theory may trace Lorentz invariance to constructive foundations - who knows? The latter scenario would certainly be an important result, but we cannot foresee the nature of future theories based on a metaphysical principle such as $P_{2}$. Thus, the judgment that without a constructive interpretation special relativity is poor and unsatisfactory from an explanatory point of view is not justified ${ }^{15}$.

\footnotetext{
${ }^{14}$ To characterize theories of principle as plainly non-explanatory is an exaggeration. To deny that they are explanatory comes down to state that thermodynamics did not render thermal phenomena more intelligible than they were before its formulation. More generally, I support a pluralistic and contextual conception of scientific explanation. I fully agree with Frisch when he states that "there may be important differences in the depth of understanding that various kinds of explanation can provide, but it seems to me to be a mistake to deny that principle or purely phenomenological theories can provide explanations at all. Rather I take it that explanation is a highly context-dependent notion and that there may even be contexts in which a phenomenological account can provide the best explanation, just as there are others in which a constructive account is called for or where an appeal to a general principle provides the simplest and best explanation" (Frisch 2011, 179-180).

${ }^{15}$ Dorato \& Felline (2010), and Felline (2011) outline an account of the explanatory power of special relativity that resembles my own. They state that Minkowski structure explains physical phenomena as a mathematical model that is independent of questions about a categorial framework - that is, the model explains independently of ontological assumptions. Now, they assert that special relativity as a theory of principle explains structurally (in terms of models) (Dorato \& Felline 2010, 198; Felline 2011, sections 2 and 3). I disagree: special relativity, in its 1905 principle-formulation, explains neither structurally nor chronogeometrically, but its Minkowski-spacetime formulation does. Dorato and Felline do not consider that the analytic connection between Lorentz invariance and Minkowski structure had to be unfolded in order to enhance the explanatory power of Einstein's theory.

Van Camp (2011) also refers to Disalle's arguments to characterize the explanatory nature of special relativity. However, he does not have in mind a properly chronogeometric explanation. Based on Disalle's views, he argues that special relativity belongs to a class of theories "that serve as preconditions for the possibility of scientific knowledge by establishing a consistent conceptual framework that defines the meaning of empirical investigations under it" (1104). Now, rather than assigning this Kantian-like constitutive role to Minkowski structure, he assigns it primarily to Einstein's principles-that is, he also misses the relevance of the analytic connection. Furthermore, since Einstein's principles, that play the Kantian-like constitutive-explanatory role, determine relativistic kinematics, Van Camp (1106-1107) takes sides with Janssen in that relativistic kinematics are more fundamental than Lorentz invariant dynamics. That is, he accepts that there is an arrow of explanation from Minkowski spacetime to Lorentz invariance.
} 


\section{CONCLUDING REMARKS}

I have argued that the Janssen-Brown debate relies on a misleading conception of the relation between Minkowski spacetime structure and Lorentz invariance. To state that there is an arrow of constructive explanation between them, regardless of its direction, results in that controversial issues concerning the ontological status of spacetime get involved in an interpretive question about the foundations of the theory. Furthermore, the debate is also undermined by an unjustified demand for a constructive version of Einstein's theory. Thus, concerning Lorentz invariance and Minkowski spacetime, to ask which is the horse and which is the cart constitutes a debate that sheds more obscurity than light on the issue of the explanatory nature of special relativity.

I claim that a much more natural reading of the relevance of Minkowski's work with respect to Einstein's original formulation of the theory consists in the uncontroversial thesis that the German mathematician simply unfolded, in precise and complete terms, the chronogeometric structure underlying Einstein's 1905 paper. In simple words, Minkowski structure and Lorentz invariance are like the two sides of a single coin. To look for an explanatory arrow between them constitutes an overinterpretation, for the arrow is better conceived as bidirectional and analytic. This proposal also allows a more nuanced interpretation of the explanatory power and nature of special relativity: Minkowski's display of the spatiotemporal structure posited by the theory contributes to make physical phenomena intelligible by means of chronogeometric explanations that add up to the explanations of principle that the theory, in its 1905 formulation, offers.

The view I am proposing here is not affected by the problems underlying the Janssen-Brown debate that I pointed out in section 4 . The description of the explanatory power of special relativity in terms of chronogeometric and of principle explanations does not endorse the unjustified urgent demand for a constructive version of the theory, and it clarifies that the lack of such a version does not render it explanatorily unsatisfactory. This statement, I must say, assumes a pragmatic-pluralistic view of the concepts of scientific explanation and scientific understanding - a view which, I think, constitutes the appropriate lesson we should draw after decades of discussion about these matters.

On the other hand, the statement that the explanatory load carried by Minkowski spacetime is given by chronogeometric explanations is not committed to a specific position concerning spacetime ontology. Recall that the explanatory work is performed by Minkowski structure taken as the conceptual scaffolding postulated by the theory. This view requires neither that we postulate that spacetime is a self-standing entity, nor that we endorse a form of eliminative relationism. The explanatory power of Minkowski spacetime structure does not rely on ontological issues: the type of explanation involved is chronogeometric, not constructive, so it does not involve a causal-ontic import. In short, Minkowski structure does not require an ontological interpretation in order to provide chronogeometric explanations.

Now, that my construal of the explanatory foundations of special relativity in terms of chronogeometry does not require an ontological commitment towards spacetime can be taken as a motivation to reject substantivalism (absolutism) - appealing to Ockham's principle, for example. Explanatory issues pervade the substantivalism/relationism debate: self-standing spacetime is posited as a way to explain basic physical features, and it is rejected by relationists, in part, because they claim that it is explanatorily unnecessary. However, my main point here is that my proposal is not necessarily committed either to substantivalism (as Janssen's interpretation) or to relationism (as Brown's). One could accept the epistemological description of chronogeometric explanations presented, and still support the ontological substantivalist (absolutist) thesis that spacetime is a self-standing entity or a self-standing structure of relations. This would probably be a rather unmotivated form of substantivalism (unless some other role is 
assigned to self-standing spacetime), but it is a logically consistent stance. The same holds for relationism. One can accept my epistemological thesis and support the ontological thesis that spacetime reduces to the relations between physical objects ${ }^{16}$. The important point is that they are logically independent theses. Furthermore, one could endorse my arguments and evaluate the substantivalism/relationism debate as a futile, or even meaningless, dispute (just for the record, this is, more or less, my own opinion). Thus, ontological considerations about Minkowski chronogeometric structure do not play a role in my construal of the explanatory foundations of special relativity.

To conclude, let me reevaluate some points in Janssen's and Brown's positions. On the one hand, I agree with Janssen in that the explanation that Minkowski's formulation provides for relativistic effects (not for Lorentz invariance) consists in that they are manifestations of default spatiotemporal behavior of physical systems. Actually, this statement grasps the epistemic content of chronogeometric explanations quite neatly-unfortunately, it is introduced in the overinterpretive terms of the debate. I do not agree with Janssen in that Minkowski spacetime explains Lorentz invariance, for they are analytically connected. I do not agree either with Janssen's view that Minkowski structure, in order to be explanatory, has to be understood as the reality behind the phenomena. Once again, in order to make relativistic phenomena scientifically intelligible - in Disalle's sense-by conceiving them as manifestations of the standard spatiotemporal behavior encoded in Minkowski structure, it is not necessary to postulate spacetime as a self-standing entity or structure of relations. We get a chronogeometric explanation simply by understanding Minkowski spacetime structure as a conceptual scaffolding - no ontological status is assumed - that renders relativistic effects intelligible. This is certainly more than a mere explanation of principle, for Einstein's two principles and the Lorentz transformations are not able to offer, in and by themselves, a chronogeometric explanation of relativistic phenomena, the analytic link between Lorentz invariance and Minkowski structure is needed. The chronogeometric characterization of the explanatory power of special relativity is not constructive either. If (as Janssen) we want an explanation of this kind based on Minkowski structure, we must assume a form of spacetime absolutism - but special relativity, as a physical theory, is agnostic about the ontology of spacetime.

Turning now to a reevaluation of Brown's view, let us recall that the demand for a constructive interpretation of special relativity can be understood in the light of principles $P_{1}$ and $P_{2}$. I argued that $P_{2}$ is a metaphysical principle whose truth-value cannot be determined. However, we can consider it from a different point of view: instead of true, we may deem it as useful and fruitful. That is, rather than as a metaphysical principle, we can interpret it as a pragmatic and heuristic principle. Accordingly, Brown's arguments can be understood in a weaker way, that is, not as pointing out to an alleged explanatory gap in special relativity that needs to be filled with a constructive version of the theory, but as an expression of an epistemological commitment towards $P_{2}$ as a heuristic principle. Thus, we can accept the description of the epistemic power of special relativity in terms of chronogeometric explanations, and, at the same time, to keep an open mind about the possibility that a fundamental (quantumgravity) theory may provide for further dynamical-constructive foundations. Lorentz invariance and

\footnotetext{
${ }^{16}$ Anyway, it should be a more nuanced form of relationism. If one follows Disalle's account of the explanatory power of Minkowski structure, one cannot accept the hard-nosed relationist thesis that space and time are abstractions performed on top of objective and directly observed relations between physical objects. Disalle states that "what the standard epistemological view failed to consider, from Leibniz and Mach through Einstein and his followers, was that the "observable relations" themselves are theoretical objects. Even a purely kinematical description of the relative motion of bodies incorporates theoretical assumptions [...]. The "observable relations", in other words, involve theoretical claims about space, time, and motion and the ways in which they are interconnected [...]. Observers don't simply observe the objective relations and then hypothesize the existence of spacetime to explain them" (Disalle 1995, 319-320).
} 
Minkowski structure may be ultimate, brute cornerstones of physics, but we cannot discard the possibility that they may be founded in constructive terms by a future theory ${ }^{17}$. Now, this open-minded attitude does not require that we go back to a conception of the connection between Minkowski structure and Lorentz invariance in terms of an arrow of explanation. Rather, if it were the case that a fundamental theory unravels the constructive foundations of relativistic phenomena, that would count as an explanation, in one fell swoop, of both Lorentz invariance and Minkowski spacetime structure-because they are analytically connected. Besides, these eventual constructive foundations would not come to solve an explanatory deficiency in Einstein's theory, but they would provide us with deeper insight. Thus, this weaker reading of Brown's proposal is coherent with my arguments concerning the explanatory nature of special relativity and the connection between Minkowski structure and Lorentz invariance.

\section{ACKNOWLEDGMENTS}

I thank an anonymous referee and Dennis Dieks for their helpful comments on an earlier version of this article. I also thank the financial support provided by Research Project VRIEA-PUCV 039.369/2016, and by FONDECYT Grant 1150661.

\section{REFERENCES}

Acuña, P. (2014). “On the Empirical Equivalence between Special Relativity and Lorentz's Ether Theory". Studies in History and Philosophy of Modern Physics, 46: 283-302.

Balashov, Y. \& Janssen, M. (2003). "Presentism and Relativity", British Journal for the Philosophy of Science, 54: 327- 346.

Brown, H. (2003). "Michelson, Fitzgerald and Lorentz: the origins of special relativity revisited", Bulletin de la Société des Sciences et des Lettres de Lódz, Volume LIII; Série: Reserches sur les Déformations, XXXIX: 2335.

Brown, H. (2005a). Physical Relativity: space-time structure from a dynamical perspective. Oxford: Oxford University Press.

Brown, H. (2005b). “Einstein's misgivings about his 1905 formulation of special relativity". European Journal of Physics, 26: S85-S90.

\footnotetext{
${ }^{17}$ There is a passage in Janssen (2009) that comes close to this point: "It does not follow from any of this that it is fruitless to ask why space-time is Minkowskian and to search for a deeper explanation of Lorentz invariance and other features it encodes [...]. We can imagine that Minkowski space-time will emerge in the low-energy limit of some future theory of quantum gravity that does not include any spatio-temporal notions among its basic concepts. Such a theory would provide an answer to the question "Why Minkowski space-time?" There is no reason to think, however, that this deeper theory would require us to move any of the phenomena in which Lorentz invariance manifests itself from the column of kinematics to the column of dynamics as established by special relativity" (Janssen 2009, 49-50). That is, even if such a theory were available, phenomena in which Lorentz invariance is involved would still be examples of default spatiotemporal behavior-we could still explain them in chronogeometric terms-but the new theory would provide something else: a constructive explanation for that behavior.
} 
Brown, H. \& Pooley, O. (2001). “The Origin of the Spacetime Metric: Bell's 'Lorentzian pedagogy' and its significance in general relativity", in C. Callender \& N. Huggett (Eds.) Physics Meets Philosophy at the Planck Scale: contemporary theories in quantum gravity, 256-274. Cambridge: Cambridge University Press.

Brown, H. \& Pooley, O. (2006). "Minkowski Space-Time: a glorious non-entity", in D. Dieks (Ed.) The Ontology of Spacetime, 67-89. Amsterdam: Elsevier.

Butterfield, J. (2007). "Reconsidering Relativistic Causality". International Studies in Philosophy of Science, 21: 295-328.

Disalle, R. (1994). "On Dynamics, Indiscernibility, and Spacetime Ontology", British Journal for the Philosophy of Science, 45: 265-287.

Disalle, R. (1995). “Spacetime Theory as Physical Geometry". Erkenntnis, 42: 317-337.

Disalle, R. (2006). Understanding Spacetime: the philosophical development of physics from Newton to Einstein. Cambridge: Cambridge University Press.

Dorato, M. (2000). “Substantivalism, Relationism, and Structural Spacetime Realism". Foundations of Physics, 30: 1605-1628.

Dorato, M. \& Felline, L. (2010). "Structural Explanations in Minkowski Spacetime: which account of models?", in V. Petkov (Ed.) Space, Time and Spacetime: physical and philosophical implications of Minkowski's unification of space and time, 181-192. Amsterdam: Springer.

Einstein, A. (1905). “On The Electrodynamics of Moving Bodies", in J. Stachel (Ed.) (2005), Einstein's Miraculous Year: five papers that changed the face of physics, 123-160. Princeton: Princeton University Press.

Felline, L. (2011). "Scientific Explanation between Principle and Constructive Theories", Philosophy of Science, 78: 989-1000.

Frisch, M. (2011). "Principle or Constructive Relativity", Studies in History and Philosophy of Modern Physics, 42: 176-183.

Friedman, M. (1983). Foundations of Spacetime Theories: relativistic physics and philosophy of science. Princeton: Princeton University Press.

Hagar, A. \& Hemmo, M. (2013). "The Primacy of Geometry", Studies in History and Philosophy of Modern Physics, 44: 357-364.

Hoving, D. (2013). "Matter or Geometry as Fundamental in Relativity Theory: how not to teach special relativity". http://philsci-archive.pitt.edu/9737/.

Janssen, M. (1995). A Comparison between Lorentz's Ether Theory and Special Relativity in the Light of the Experiments of Trouton and Noble. Dissertation.

Janssen, M. (2002a). "Reconsidering a Scientific Revolution: the case of Einstein versus Lorentz", Physics in Perspective, 4: 421-446.

Janssen, M. (2002b). "COI Stories: explanation and evidence in the history of science", Perspectives on Science, 10: 457-522. 
Janssen, M. (2009). "Drawing the Line between Kinematics and Dynamics in Special Relativity", Studies in History and Philosophy of Modern Physics, 40: 26-52.

Mermin, D. (2005). It's About Time: understanding Einstein's relativity. Princeton: Princeton University Press.

Miller, D. (2010). "A Constructive Approach to the Special Theory of Relativity", American Journal of Physics, 78: 633-638.

Minkowski, H. (1909). "Space and Time", in Lorentz, H. et al. (2008), The Principle of Relativity, 73-91. New York: Dover.

Norton, J. (2008). "Why Constructive Relativity Fails", British Journal for the Philosophy of Science, 59: 821834.

Nerlich, G. (1976). The Shape of Space. Cambridge: Cambridge University Press.

Nerlich, G. (2010). "Why Spacetime Is Not a Hidden Cause: a realist story”, in V. Petkov (Ed.) Space, Time and Spacetime: physical and philosophical implications of Minkowski's unification of space and time, 181192. Amsterdam: Springer.

Pauli, W. (1921) [1981]. Theory of Relativity. New York: Dover.

Sklar, L. (1995). Physics and Chance: philosophical issues in the foundations of statistical mechanics. Cambridge: Cambridge University Press.

Slowik, E. (2005). "Spacetime, Ontology, and Structural Realism". International Studies in the Philosophy of Science, 19: 147-166.

Van Camp, W. (2011). “On Kinematic versus Dynamic Approaches to Special Relativity”. Philosophy of Science, 78: 1097-1107. 